\title{
A termografia infravermelha na avaliação dos pontos-gatilho miofasciais em patologias do ombro
}

\section{Infrared thermography in evaluation of myofascial trigger points in the shoulder pathologies}

\author{
Camila Rossili Eduardo Sehnem²; Claudete Rempel ${ }^{3}$ \\ ${ }^{1}$ Fisioterapeuta - Centro Universitário - Univates. Lajeado, RS - Brasil. \\ ${ }^{2}$ Fisioterapeuta, Mestre em Ambiente e Desenvolvimento - Centro Universitário - Univates. Lajeado, RS - Brasil \\ ${ }^{3}$ Bióloga, Doutora em Ecologia - Centro Universitário - Univates. Lajeado, RS - Brasil. \\ Endereço para correspondência \\ Camila Rossi \\ Av. Alberto Pasqualini, 2350, Universitário \\ 95900-000 - Lajeado - RS [Brasil] \\ camilarossif@gmail.br
}

Resumo

Introdução: Os pontos-gatilho miofasciais são bandas tensas perceptíveis à palpação. Eles podem ser de origem espontânea ou causados por estímulos mecânicos, podendo desencadear dor local e referida em sítios próximos ou distantes. Objetivo: Neste estudo, busca-se analisar incidência, sensibilidade e localização de pontos-gatilho miofasciais em pacientes com diagnóstico clínico de bursite ou tendinite em ombro, por meio da termografia infravermelha. Método: Foram avaliados 11 indivíduos, com média de idade de 40,8 (11,5) anos, utilizando-se algometria de pressão e termografia. Resultados: Todos os sujeitos avaliados apresentaram pontos-gatilho miofasciais em alguns dos músculos abordados, sendo estes o trapézio superior, elevador da escápula, supraespinhal, infraespinhal e romboides. Conclusão: Foi possível verificar a importância da termografia infravermelha como um componente auxiliar na avaliação dos pontos-gatilhos miofasciais.

Descritores: Bursite; Pontos-gatilho; Tendinite; Termógrafo.

\begin{abstract}
Myofascial trigger points (TrPts) are tense, palpable bands. They can arise spontaneously or as a result of mechanical stimuli, and are capable of setting off local pain that is reported by subjects in proximal or distant areas. Objective: This study aimed to analyze the incidence, sensitivity and localization of the TrPts in patients with clinic diagnosis of bursitis and shoulder tendinitis by means of infrared thermography. Method: Eleven subjects with mean age of 40.8 (11.5) years were assessed using algometry and thermography. Results: All subjects showed myofascial trigger points in some muscles, as upper trapezius, levator scapulae, supraspinatus, infraspinatus and rhomboids. Conclusion: It was possible to verify the relevance of infrared thermography as a complementary component in the myofascial trigger points assessment.
\end{abstract}

Key words: Bursitis; Tendinitis; Thermograph; Trigger points. 


\section{Introdução}

Os pontos-gatilho (Pg) miofasciais são pequenas áreas hipersensíveis localizadas nas bandas musculares tensas palpáveis do músculo esquelético. Eles podem ser de origem espontânea e/ou ser causados por estímulos mecânicos, desencadeando dor local e referida em áreas distantes ou próximas ${ }^{1}$.

Conforme Asher ${ }^{2}$, os pontos-gatilho miofasciais podem variar de tamanho - de uma pequena protuberância até um grande inchaço -, e ser notados na superfície do corpo, entremeados nas fibras musculares.

Os Pgs, que acarretam como queixa clínica a dor, são facilmente identificados, quando digitalmente comprimidos.

Os pacientes com Pgs miofasciais ativos, em geral, reclamam de dor constante e mal localizada, regional e nos tecidos subcutâneos, incluindo músculos e articulações. Eles raramente se queixam de dor aguda, tipo cutânea, em local específico. A dor miofascial costuma ser referida a certa distância do Pg, em um padrão característico a cada tipo de músculo.

O músculo trapézio superior é comumente afetado em decorrência de um trauma agudo, tensionado por uma lesão crônica ou agredido por movimentos repetitivos. Também pode desencadear, devido ao padrão adotado, momentos de angústias/ansiedade ou dor. Quando digitalmente pressionados, os Pgs provocam dor referida na região cervical, ao occipúcio e à têmpora ${ }^{1}$. Conforme Simons, Travell e Simons ${ }^{1}$, os sintomas gerados por um Pg no trapézio superior podem ser pertinentes e similares a disfunções articulares abaixo das vértebras C2, C3 e C4.

A tensão postural gera um "pescoço rígido", acometendo o músculo elevador da escápu$\mathrm{la}^{3}$. Com isso, os pacientes queixam-se de dor na região do ângulo cervical dessa parte do corpo e do pescoço rígido doloroso.

Quando um paciente relata sentir uma dor profunda na parte anterior do ombro, pode estar relacionada ao músculo infraespinhal ${ }^{4}$. Asher ${ }^{2}$ relata que a dor referida do músculo infraespinhal acomete as proximidades da cabeça longa do bíceps braquial, com irradiação no ventre do bíceps, apresentando sintomas difusos na distribuição de inervação do nervo mediano.

A presença de um Pg no músculo supraespinhal causa uma dor referida local ou intensa na parte lateral do braço, região média do deltoide. Também se estende ao longo do braço, acometendo o epicôndilo lateral do cotovelo. A sensibilidade à dor que esse desencadeia pode ser confundida facilmente com bursite subdeltoidea ${ }^{1}$. A existência de um ponto-gatilho miofascial em um músculo que, devido à palpação, procede em sensibilidade local intensa e com irradiações da dor em regiões distintas para cada ponto pode ser definida com uma síndrome dolorosa de tecidos moles, ou seja, a síndrome dolorosa miofascial ${ }^{5}$.

De acordo com Yeng e Kaziyama ${ }^{6}$, a palpação do ponto-gatilho pode reproduzir a dor, descrita pelo paciente, característica à sua patologia, dor referida para músculos, tendões, fáscias e cápsulas articulares.

Dentre os achados físicos por meio da palpação manual, será possível observar uma banda tensa com um nódulo sensível. E esse ponto, quando submetido a uma pressão, o paciente irá reconhecer a dor referida como a típica sentida em seu dia a dia. Mas, além da dor, pode desencadear outras alterações sensoriais referidas, bem como sensibilidade e disestesias.

A tensão e/ou sobrecarga muscular pode afetar a musculatura adjacente e intensificar o quadro álgico. A presença dos pontos-gatilho miofasciais na cintura escapular e cervical pode interferir no surgimento de dor referida para o membro superior em pacientes portadores de patologias ortopédicas e traumatológicas. As patologias comumentes encontradas são as tendinites e bursite de ombro.

Trabalhos recentes demonstram o uso da termografia computadorizada como um artifício complementar favorável para o diagnóstico e avaliação dos pontos-gatilho miofasciais ${ }^{7}$. Outros estudos também apresentam a termo- 
grafia infravermelha como método eficiente na complementação diagnóstica de afecções de outros tecidos, por exemplo, bursa, tendões, tecido ósseo, até mesmo, tumores malignos ${ }^{7,8}$. Contudo, ainda percebe-se uma carência de estudos utilizando a termografia como recurso de complementação do diagnóstico fisioterapêutico.

Segundo Brioschi et al. ${ }^{9}$, o termógrafo é um dispositivo que capta a radiação infravermelha, emitida em todos os corpos acima do zero absoluto. Essa radiação é transformada em uma imagem, caracterizada por um espectro de diferentes cores, variáveis, conforme a temperatura relativa de cada região corporal. No caso dos pontos-gatilho miofasciais, estes apresentam-se como uma superfície de hiper-radiação de calor, com propagação do sinal ao longo do local de dor referida.

O uso da algometria de pressão na avaliação dos pontos-gatilho miofasciais associada com a imagem infravermelha tem-se mostrado uma correlação estatisticamente significativa, segundo estudos de Fischer ${ }^{10}$. A algometria vem sendo utilizada para fornecer diagnósticos, experimentais e médico-legais. Ela é usada como um método quantitativo para estes, que busca mensurar a intensidade da dor e também a localização dos pontos dolorosos ${ }^{11}$.

A algometria baseia-se em princípios físicos que geram a dinâmica das forças aplicadas à superfície, assim mensurando a encargo dos nociceptores à pressão aplicada sobre eles. Os resultados são expressos em $\mathrm{kg} / \mathrm{cm}^{2}{ }^{12}$.

$\mathrm{Na}$ área da fisioterapia, é importante a detecção dos pontos-gatilho miofasciais, pois auxilia no tratamento fisioterapêtico. Alguns deles podem desencadear dor referida, que, muitas vezes, é associada pelo paciente como sua queixa principal 5 .

Dessa forma, neste estudo, busca-se analisar a presença e sensibilidade dos pontos-gatilho miofasciais em pacientes com patologias no ombro, como a bursite e a tendinite, além de verificar o mapeamento destes e suas regiões de irradiação.

\section{Material e método}

Otrabalho foi aprovado peloComitêde Ética em Pesquisa do Centro Universitário Univates (COEP), $n^{\circ}$ do protocolo 75939/20/08/2012.

Foram avaliados 11 pacientes em duas clínicas de fisioterapia. Os voluntários deveriam se enquadrar nos critérios de inclusão, sendo estes, apresentar como diagnóstico clínico tendinite ou bursite de ombro e ter assinado o Termo de Consentimento Livre e Esclarecido; e, como critério de exclusão, não fazer uso de medicamentos e não estar afastado do trabalho. Para obter fidedignidade dos resultados, a coleta de dados foi realizada em uma sala climatizada a $20^{\circ} \mathrm{C}$, a fim de eliminar possíveis artefatos no sinal. Além disso, foram dadas as seguintes orientações aos participantes: evitar atividade física ou fisioterapia no mínimo uma hora antes da avaliação, não usar roupas apertadas para evitar compressões nos tecidos. A imagem infravermelha foi obtida com o dispositivo posicionado a um metro de distância do voluntário ${ }^{9}$. Foram captadas imagens em vista anterior, posterior e perfil, do lado em que o participante relatava sua queixa. Após, foi realizada a palpação dos músculos trapézio superior, elevador da escápula, supraespinhal e infraespinhal e, se localizado um ponto-gatilho miofascial, era aplicado o algômetro de forma perpendicular ao ponto doloroso para verificar a sensação de dor desse local à pressão. A sensibilidade dolorosa de cada ponto será apresentada em valor médio por músculo. Nessa etapa, o participante deveria indicar o momento em que percebia tal sensação, diante da compressão pelo dispositivo. A análise dos dados foi realizada por intermédio do Microsoft Windows Excel 2010®; e os dados, apresentados na forma de média (desvio-padrão). Para comparação da diferença entre a visibilidade dos pontos-gatilho na imagem infravermelha, foi aplicado o Teste do Sinal ${ }^{13}$, sendo consideradas significativas as diferenças maiores do que 5\%. As imagens foram analisadas pelo software FLIR tools, permitindo o tratamento da imagem, e os contrastes necessários para a visualização. 


\section{Resultados e discussão}

Para a obtenção dos resultados, foram avaliados 11 pacientes, sendo nove do sexo feminino $(81,9 \%)$, e dois, do masculino $(18,1 \%)$, com idade média de 40,8 $(11,5)$ anos, apresentando como diagnóstico clínico bursite ou tendinite em ombro.

Todos os pacientes investigados no estudo apresentaram ponto-gatilho miofascial em alguns dos músculos avaliados, sendo estes o trapézio superior, elevador da escápula, supraespinhal, infraespinhal e romboides. Do total da amostra, nove tinham ponto-gatilho no músculo trapézio superior $(81,8 \%)$, sendo visível na termografia em seis $(66,6 \%)$. No elevador da escápula, cinco participantes mostraram ponto-gatilho, visível na termografia infravermelha em apenas dois casos (40\%). No músculo supraespinhal, houve presença de ponto-gatilho em dois, sendo ambos visíveis na termografia $(100 \%)$. No músculo infraespinhal, cinco mostraram pontos-gatilhos, sendo visíveis na imagem infravermelha nos cinco casos $(100 \%)$. E nos romboides, os pontos-gatilhos foram visíveis na imagem infravermelha nos três casos avaliados que apresentaram pontos-gatilho miofasciais $(100 \%)$, conforme mostra o gráfico na Figura 1.

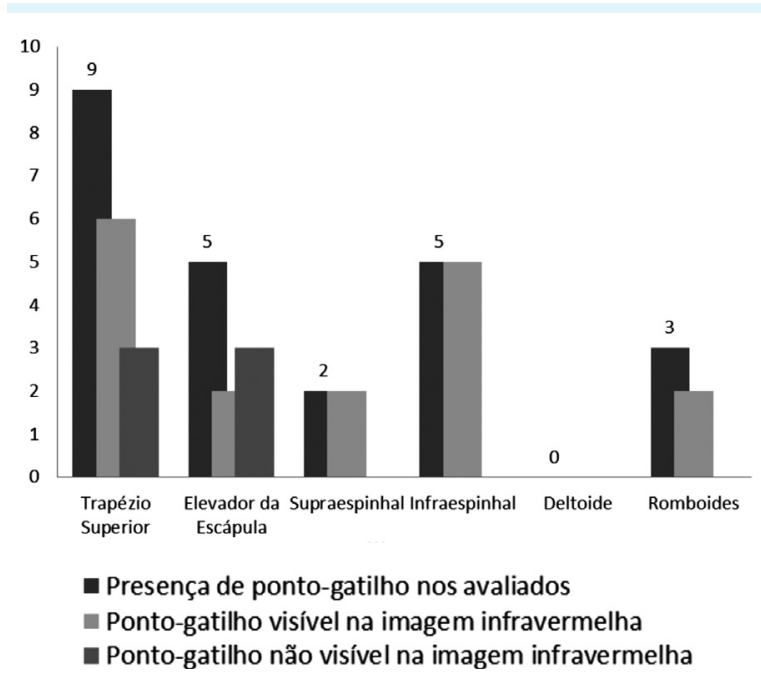

Figura 1: Relação da identificação dos pontos-gatilho
Os resultados supracitados corroboram os de Simons, Travell e Simons ${ }^{1}$, que caracterizam o músculo trapézio superior como um dos mais acometidos por pontos-gatilhos miofasciais. Sola e Kuitert ${ }^{14}$, em um estudo, relataram que, quando um paciente possui dor na região do pescoço e do ombro, os músculos elevador da escápula e infraespinhal estão diretamente relacionados com a aparição dos pontos-gatilho no trapézio superior. Quando um Pg se torna crônico, esse local de tensão pode converter-se em uma região hiporradiante ${ }^{15}$, dificultando a identificação do ponto-gatilho na imagem infravermelha. No estudo realizado, a visualização dos pontos-gatilho miofasciais no músculo trapézio superior não foi aparente como área de hiper-radiação na imagem infravermelha em três casos avaliados. Na palpação manual, foi possível identificar uma banda tensa muscular, apresentando-se como uma região hipersensível. Os músculos do manguito rotador (supra e infraespinhoso), que possuem função estabilizadora dessa região ${ }^{16}$, diante de uma patologia do ombro, estão suscetíveis a apresentar pontos de hiper-radiação e, devido a sua localização superficial, é fácil identificar e a visualizar os pontosgatilhos miofasciais.

Entre os 11 pacientes avaliados apresentando pontos-gatilho miofasciais ativos, pode-se observar um limiar de dor semelhante na aplicação da algometria, tendo média de $2,54 \mathrm{~kg} / \mathrm{cm}^{2}$ (Figura 2).

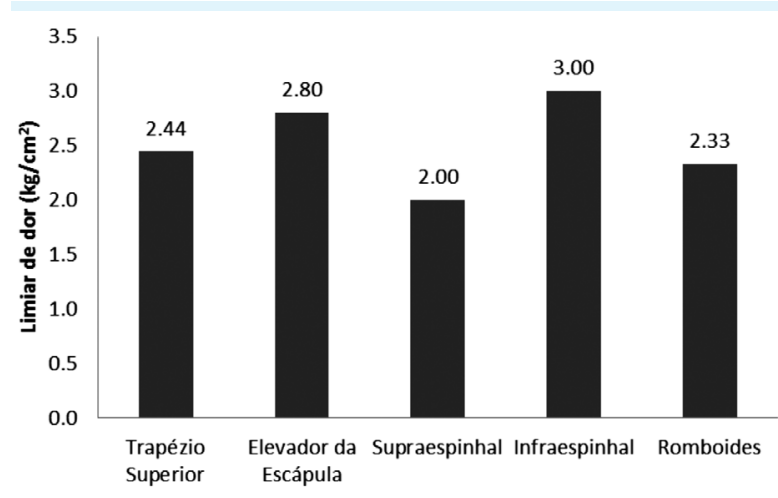

Figura 2: Média de limiar de dor $\left(\mathrm{em} \mathrm{kg} / \mathrm{cm}^{2}\right)$ nos músculos avaliados pela algometria 
Houve diferença estatística significativa $(\mathrm{p}<0,05)$ apenas para os pontos infraespinhais entre a visibilidade na imagem do ponto e a não visibilidade.

Conforme Hudson ${ }^{17}$, a dor é um mecanismo de defesa contra agentes agressores internos ou externos. Os nociceptores detectam, a partir de um neurônio primário, os estímulos dolorosos, e estes neurônios reproduzem estímulos físicos (pressão, calor e frio) e químicos (ácidos, substâncias irritantes). A percepção a um estímulo externo é um episódio que irá produzir uma sensibilidade, sendo esta controlada ou não.

Os nociceptores determinam a tolerância dolorosa e o limiar de percepção, que está diretamente relacionado com a capacidade de captação nociceptiva, dos controles excitatórios superiores e indiretamente relacionado com os controles inibitórios superiores. Portanto, se houver um desequilíbrio entre esses centros, estímulos subliminares poderão produzir uma percepção dolorosa (alodínia), mas também poderão acarretar tolerância diminuída a estímulos dolorosos (hiperalgesia) $)^{18,19}$.

Conforme Simons, Travell e Simons ${ }^{1}$, a presença de pontos-gatilho miofasciais em músculos que apresentam localização mais superficial é facilmente identificada e exibe uma maior sensibilidade à palpação. $O$ estudo realizado demonstrou que os pontos-gatilho miofasciais nos músculos supraespinhal, infraespinhal e romboides são identificados com facilidade à palpação manual, sendo visíveis na imagem infravermelha em todos os casos.

Um ponto-gatilho na região do elevador da escápula pode ocasionar outro na região do músculo infraespinhal. Um estudo, realizado por Sola e Kuitet ${ }^{14}$, demonstra que, em $55 \%$ dos avaliados, a presença do ponto-gatilho no infraespinhal é secundária ao elevador da escápula. $\mathrm{Na}$ pesquisa aqui apresentada, demonstra-se a relação da presença e da sensibilidade dos pontosgatilho dos músculos elevador da escápula para o infraespinhal em 100\% dos casos analisados.

Em um estudo, Fischer e Chang ${ }^{20}$ concluíram que a hiper-radiação em um ponto-gatilho mio- fascial não precisa ser necessariamente por condução, e sim, por uma resposta reflexa medular somatocutânea, sucedendo a uma vasodilatação da derme bloqueando receptores alfa-adrenérgicos por meio de impulsos nociceptivos locais dos pontos-gatilho, acedidos por contratura, isquemia e liberação de substâncias algogênicas.

Para Brioschi et al. ${ }^{9}$, os pontos-gatilho são sítios de hiperirritabilidade com um feixe muscular tenso localizado; por isso, aparecem em áreas aquecidas hiper-radiantes.

A detecção dos pontos-gatilho miofasciais pela termografia mostra-se efetiva. No caso mostrado na Figura 3, nota-se uma região de maior irradiação média do músculo deltoide, sendo esta, conforme Asher², uma das áreas de irradiação de um ponto-gatilho miofascial do supraespinhal. Pode-se também atribuir a hiper-radiação em região do deltoide como uma manifestação secundária a uma alteração dos tecidos profundos da região, como a bursa ou algum tendão do manguito rotador.

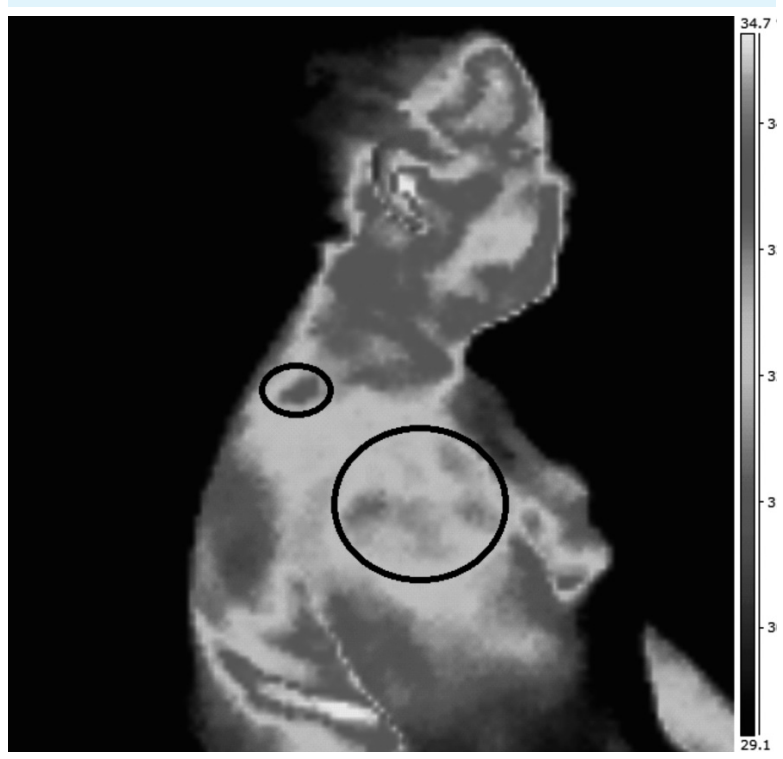

Figura 3: Hiper-radiação sobre o pontogatilho no músculo supraespinhal, com hiper-radiação reflexa para a região média no músculo deltoide

Observa-se área de irradiação na região lateral do braço, possivelmente causada pela presença de um ponto-gatilho miofascial no 
músculo supraespinhal, no participante que apresenta, como diagnóstico clínico, tendinite do tendão de bíceps. Também pode-se perceber pequena área de hiper-radiação no redondo menor, sendo também referida dor na parte lateral do braço, presente com frequência em pacientes com diagnóstico clínica de tendinite no tendão do bíceps².

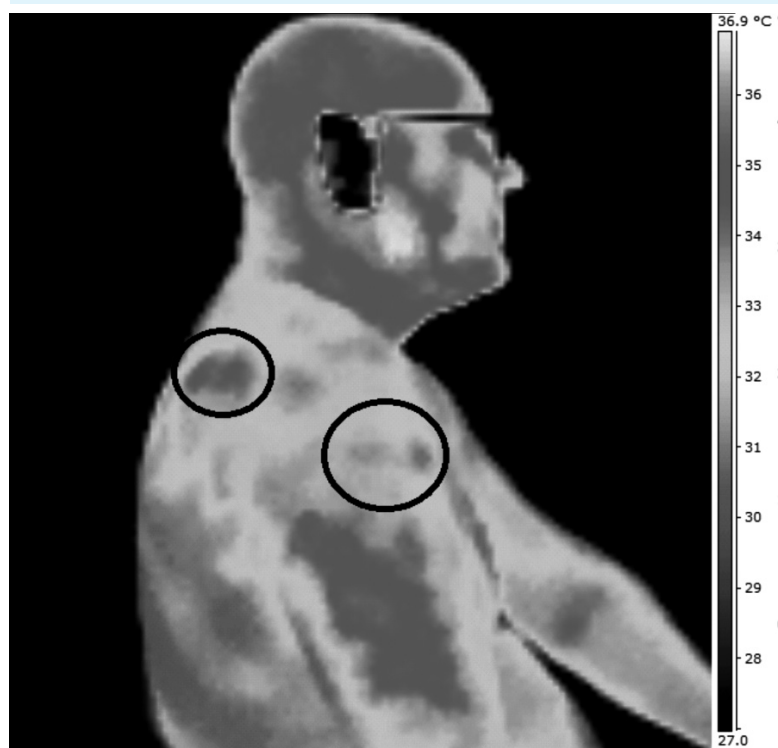

Figura 4: Hiper-radiação sobre o pontogatilho no músculo supraespinhal, com hiper-radiação reflexa para a região média no deltoide e no braço

Na Figura 5, nota-se uma área de hiper-radiação predominante no lado esquerdo, e outra, na região do músculo infraespinhal. Percebe-se uma área elevada de hiper-radiação na cervical, com irradiação para o elevador da escápula. Conforme Kostopoulos e Rizopoulos ${ }^{21}$, a presença de um ponto-gatilho na região do elevador da escápula tem dor referida para o ângulo do pescoço, borda vertebral da escápula e parte superior do ombro.

O caso apresentado na sequência (Figura 6) mostra semelhança com o anterior (Figura 5) e relação da área de hiper-radiação da região cervical com irradiação ao elevador da escápula, atingindo o ângulo do pescoço, borda vertebral da escápula e parte superior do ombro. Percebese grande área de hiper-radiação na região de

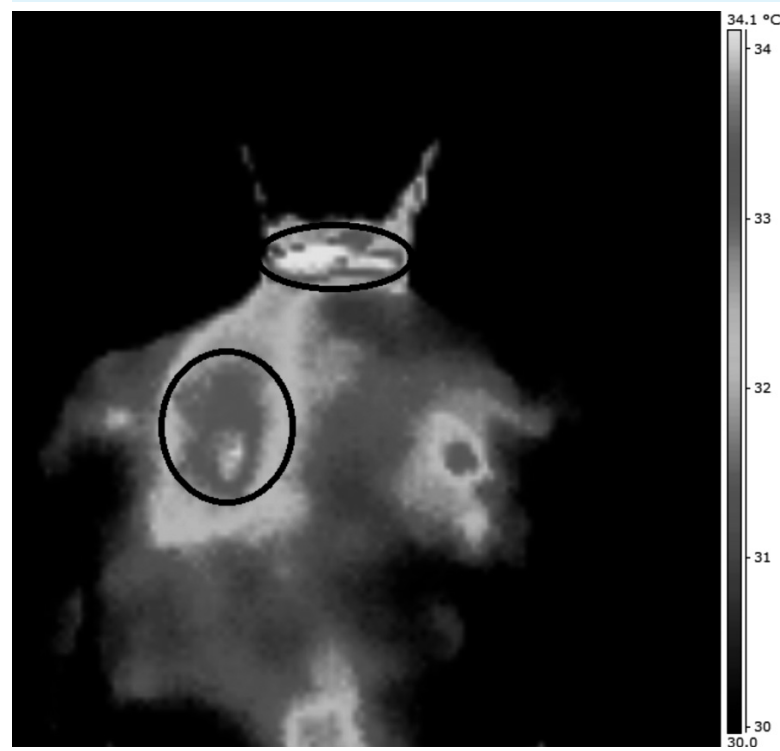

Figura 5: Hiper-radiação em região cervical, com irradiação predominante no lado esquerdo

romboides bilaterais. Pode-se arrogar que um ponto-gatilho miofascial na região de romboides está relacionado à postura antálgica que muitos pacientes adotam em razão de seu quadro álgico. Uma postura antálgica gera sobrecarga/tensão muscular, que irá diretamente provocar um encurtamento muscular, podendo, assim, causar um ponto-gatilho miofascial.

Na paciente avaliada com diagnóstico de fibromialgia apresentando bursite em ombro esquerdo, percebem-se áreas difusas de hiper-

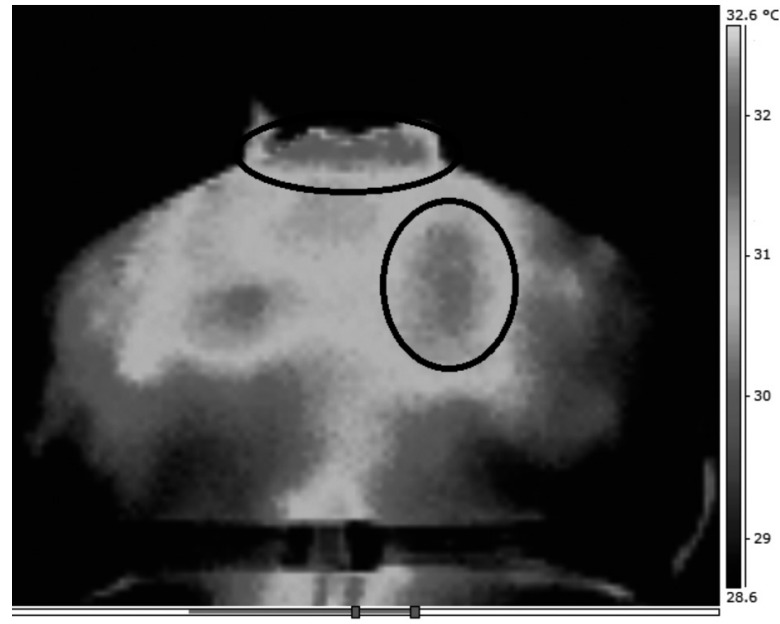

Figura 6: Hiper-radiação em região cervical, com irradiação predominante no lado direito 
radiação (Figura 7). Pacientes com diagnóstico de fibromialgia, geralmente, apresentam padrão hiper-radiante não específico, similares aos locais musculares dolorosos comumente afetados, conveniente à hipertonia muscular local; sendo diferentes de um ponto-gatilho miofascial que apresenta imagem com contornos bem determinados, regulares e elipsoide, segundo Martinez $^{22}$. Diversos autores evidenciam alterações dos mecanismos centrais do processamento da dor em pacientes portadores de fibromialgia $^{23}$. O que pode contribuir para o aumento da sensibilidade à dor, uma vez que os acometidos apresentam os tender points, que são pontos de hipersensibilidade.

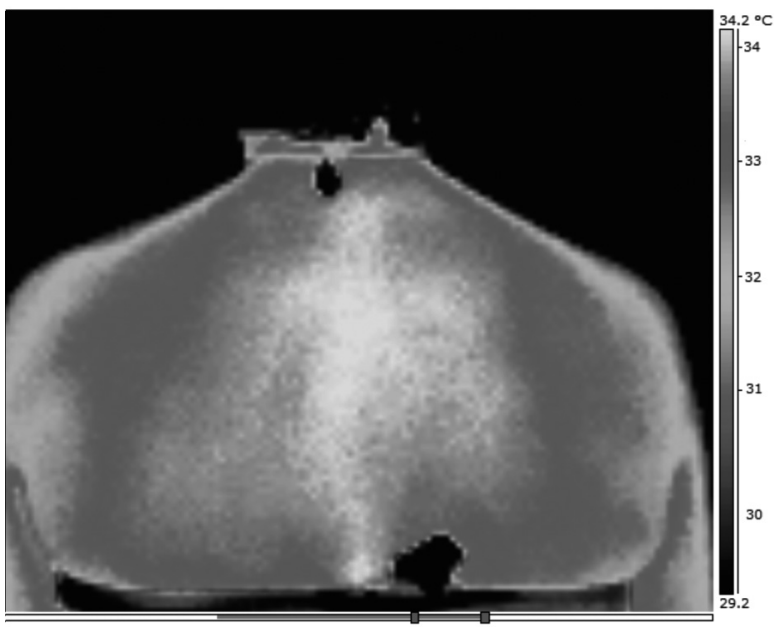

Figura 7: Paciente com fibromialgia apresentando bursite em ombro esquerdo. Áreas difusas de hiper-radiação

\section{Conclusão}

Com este trabalho, foi possível verificar a importância da termografia infravermelha como um componente auxiliar na avaliação dos pontos-gatilho miofasciais. Este dispositivo capta as áreas de maior hiper-radiação de calor, possibilitando o mapeamento dos pontos-gatilho. Observou-se que, no grupo estudado, todos os indivíduos apresentaram pontos gatilho miofasciais, sugerindo que a alteração tecidual decorrente da lesão, está associada à alteração da mio- fáscia, por meio do desenvolvimento dos pontos de tensão. Nesta pesquisa, mostrou-se a relação da palpação manual dos pontos-gatilho com a imagem infravermelha. Sendo possível também, em alguns casos, observar as regiões acometidas pela lesão, como áreas de hiper-radiação, além do local dos pontos-gatilho miofasciais. Para pacientes nessas condições, o tratamento fisioterapêutico poderá ser mais eficaz, pois será tratada a patologia específica, assim como um fator secundário que provoque dor. Sugerem-se futuros estudos longitudinais em que se explorem os potenciais da termografia infravermelha no monitoramento do processo evolutivo das patologias diante do tratamento fisioterapêutico.

\section{Referências}

1. Simons DGm Travell JG, Simons LS. Dor e disfunção miofascial: manual dos pontos-gatilho. V.1 - parte superior do corpo. $2^{\mathrm{a}}$ ed. Porto Alegre: Artmed; 2005.

2. Ascher SN. Pontos-gatilho: uma abordagem concisa. Barueri: Manole; 2008.

3. Menachin A, Kaplan O, Dekel S. Levator scapulae syndrome: an anatomic-clinical study. Bull Hosp Joint Dis. 1993;53(1):21-4.

4. Travell J. Ethyl chloride spray for painful muscle spasm. Arch Phys Med Rehabil. 1952; 33:291-8.

5. Cummings M, Baldry P. Regional myofascial pain: diagnosis and management. Best Pract Res Clin Rheumatol. 2007;21(2):367-87.

6. Yeng LY, Kaziyama HHS, Teixeira MJ. Síndrome dolorosa miofascial. Rev Med (São Paulo). 2001;80;94-110.

7. Brioschi ML, Cherem AJ, Ruiz RC, Sardá Júnior JJ, Silva FMRM. O uso da termografia infravermelha na avaliação do retorno ao trabalho em programa de reabilitação ampliado (PRA). Acta Fisiatr. 2009;16(2):87-92.

8. Pichot C. Aplicación de la termografia en el dolor lumbar crónico. Rev Soc Esp Dolor. 2001;8(2):43-7. [acesso em 2012 Nov 10]. Disponível em: <http:// revista.sedolor.es/pdf/2001_10_07.pdf >. 
9. Brioschi ML, Portela PC, Colman D, Laskawski S, Santos LC. Infrared thermal imaging in patients with chronic pain in upper limbs. J Korean Thermol. 2002;2(1):73.

10. Fischer AA. Diagnosis and management of chronic pain is physical medicine and rehabilitation. In: Ruskin - Current Therapy in Physiatry. 1984;123-45.

11. Fischer AA. Pressure threshold measurements for diagnosis of myofascial pain and evaluation of treatment results. Clin J Pain. 1987;2:207.

12. Piovesan EL, Tatsui CE, Kowacs PA, Lange MC, Pacheco C, Werneck LC. Utilização da algometria de pressão na determinação dos limiares de percepção dolorosa trigeminal em voluntários sadios: um novo protocolo de estudos. Arq Neuopsiquiatr. 2001;59(1):92-6.

13. Gomes, FP. A estatística moderna na pesquisa agropecuária. $3^{\text {a }}$ ed. Piracicaba: Potafos; 1987. 162 p.

14. Sola AE, Kuitert JH. Myofascial trigger point pain in the neck and shoulder girdle. Northwest Med. 1955;45:980-4.

15. Hakguder A, Birtane M. Efficacy of low level laser therapy in myofascial pain syndrome: and algometric and thermographic evaluation. Lasers Surg Med. 2003;33(3):339-43. [acesso em 2012 Nov 2]. Disponível em: <http://www.ncbi.nlm.nih.gov/ pubmed/14677161>.
16. Konin JG. Cinesiologia prática para fisioterapeutas. Rio de Janeiro: Guanabara Koogan; 2006.

17. Hudson AJ. Pain perception and response: central nervous system mechanisms. Can J Neurol Sci. 2000;27:2-16

18. Bindtsen L, Jensen R, Olesen J. Decreased pain detection and tolerance thresholds in chronic tension-type headache. Arch Neurol. 1996;53:373-6.

19. Duarte MA, Goulart EM, Penna FJ. Pressure pain threshold in children with recurrent abdominal pain. J Pediatr Gastroenterol Nutr. 2000;31(3):280-5.

20. Fischer AA, Chang $\mathrm{CH}$. Temperature and pressure threshold measurements in trigger points.

Thermology. 1986;1(4):212-5.

21. Kostopoulos D, Rizopoulos K. Pontos-gatilho miofasciais: teoria, diagnóstico, tratamento. Rio de Janeiro: Guanabara Koogan; 2007.

22. Martinez LM. Fibromyalgia as a sympathetically maintained pain syndrome. Curr Pain Headache Rep. 2004;8(5):385-9.

23. Branco JC, Domingues ME. Viver com a fibromialgia: a visão da doente e do médico. Lisboa: Gradiva; 2008. 